\title{
A Laser-Assisted Cellular Electrophysiology Measurement System
}

\author{
A. A. Seymen, E. Özgür, Z. Soran-Erdem, and B. Ortaç ${ }^{\circledR}$, Member, IEEE
}

\begin{abstract}
Patch-clamp technique is the gold standard for cellular electrophysiological measurements, which is capable of measuring single ion transport events across the cell membrane. However, the measurement possesses significant complexities, and it requires a high level of expertise, while its experimental throughput is nevertheless considerably low. Here, we suggest and experimentally demonstrate a laser-assisted method for performing cellular electrophysiological measurements. Femtosecond laser pulses, coupled to an optical microscope, are used to form a sub-micrometer hole on a thin polymer membrane separating two electrodes, where a nearby cell is subsequently placed onto the hole by negative pressure. Afterwards, the cell is punctured using subsequent laser exposure, revealing the cell membrane over the hole for electrophysiological recording. This system could be used to increase the output amount of the electrophysiological measurements substantially.
\end{abstract}

Index Terms-Laser nanosurgery, femtosecond laser, electrophysiology.

\section{INTRODUCTION}

B ESIDES isolating the cellular metabolism from the exterior, cell membranes gather intracellular components such as organelles and various metabolites [1]. They host membrane proteins, which correspond to $20 \sim 30 \%$ of the genes expressed in a cell [2] and are the targets for $50 \%$ of pharmaceutical drugs [3]. Although the main building block of cellular membranes are amphipathic phospholipids, which spontaneously form lipid bilayers under physiological conditions [4], membrane proteins have an important role in determining the membrane, and therefore cellular characteristics, by controlling the uptake and excretion of materials [5], transducing signals from the extracellular environment [6], communicating with other

Manuscript received September 19, 2019; revised December 28, 2020; accepted December 30, 2020. Date of publication January 5, 2021; date of current version January 18, 2021. This work was supported by the Ministry of Science, Industry, and Technology of Turkey. (Corresponding authors: E. Özgür; B. Ortaç.)

A. A. Seymen is with E-A Teknoloji LLC, 06800 Ankara, Turkey, also with the Department of Physiology, Erciyes University, 38039 Kayseri, Turkey, and also with the UNAM-National Nanotechnology Research Center, Institute of Materials Science and Nanotechnology, Bilkent University, 06800 Ankara, Turkey (e-mail: aytac@eateknoloji.com).

E. Özgür is with E-A Teknoloji LLC, 06800 Ankara, Turkey (e-mail: erol@eateknoloji.com).

Z. Soran-Erdem is with the UNAM-National Nanotechnology Research Center, Institute of Materials Science and Nanotechnology, Bilkent University, 06800 Ankara, Turkey, and also with the Department of Engineering Sciences, Abdullah Gül University, 38080 Kayseri, Turkey (e-mail: zeliha.soranerdem@agu.edu.tr).

B. Ortaç is with the UNAM-National Nanotechnology Research Center, Institute of Materials Science and Nanotechnology, Bilkent University, 06800 Ankara, Turkey (e-mail: ortac@unam.bilkent.edu.tr).

Color versions of one or more figures in this letter are available at https://doi.org/10.1109/LPT.2021.3049348.

Digital Object Identifier 10.1109/LPT.2021.3049348 cells [7], and anchoring the cell to the extracellular matrix [8]. Thus, it is not surprising that the investigation of cellular membranes is a major field of scientific research.

Among the integral membrane proteins, ion channels are particularly important because of their role in maintaining the cellular homeostasis by acting like molecular switches operating under influences such as electrical potentials [9], chemical mediators [10], mechanical [11], thermal [12], and even optical stimuli [13]. Nonetheless, their function merely depends on the basic physical laws of nature [14], [15]. Thus, ion channel research forms a basis for a wide span of disciplines investigating the physiology and pathology of the cells. The main goals of electrophysiology research are anticipating the reactions of ion channels under the influence of different stimuli, and eventually controlling them using various methods. These efforts aim not only deciphering the underlying mechanisms of their operation, but also understanding and interfering with related pathological conditions, which are essential in discovering and developing novel drugs. Considering the drawbacks of isolation problems and obtaining the same response from all isolated cells, H9c2 is a significant cell line source to investigate ion channels by electrophysiology. $\mathrm{H} 9 \mathrm{c} 2$ cells are a clonal myogenic cell line derived from embryonic rat ventricles which initially had been thought of as myocytic, but later they were realized that they possess both skeletal and cardiac muscle features [16]. In a study published by Hescheler et al., researchers demonstrated that these cells preserve several electrical and hormonal signal pathways similar to those of adult cardiac cells [17]. After this finding, H9c2 cells became the center of attraction because of their L-type channels and voltage-dependent nonspecific channels, especially for electrophysiology studies [18].

To date, patch-clamp recording is considered the gold standard for measuring the ionic currents through ion channels inside and outside the cells. This technique, which inventors are Nobel laureates, enables direct observations of ionic fluxes [19] by using finely tapered glass capillaries for electrically insulating electrodes on reciprocal sides of ion channels that seal the cell membrane by suction. Using this technique, researchers could interrogate ion channels either as constituents of a small membrane patch sealed on the tip of the glass pipette, to observe individual opening and closing of these tiny gates, or measuring the currents passing through the whole membrane. The power of this technique resides in the fact that one of the electrodes is within the capillary, while the other is in the bath solution. Therefore, as long as the fine tip of the capillary is sealed onto the cell membrane, using the electrostatic interactions among the thermally tapered glass 
and the phospholipids, the membrane patch, or the whole cell, acts analogous to a resistance-capacitance (RC) circuit, which the resistance is variable. This technique also enables the administration of different chemicals including channel blockers, forming a perfect scheme for pharmacological studies.

Besides the unprecedented opportunities provided by patchclamp measurements, this method is nevertheless one of the most cumbersome practices to apply experimentally. It requires a perfect isolation of the cells from the tissue, well-defined parameters for capillary pulling, and a complex experimental set-up. Training for patch-clamp requires considerable time for the experimenter. The throughput of the experiment is generally low. Therefore, alternative techniques that do not employ the capillary micropipettes were investigated for simplifying the process. Planar patch-clamp systems, for instance, gained considerable reputation regarding their straightforward use. Instead of using capillaries, these systems utilize chips that have a micron-scale hole, where the cells are pulled by suction [20]. There are automated systems using many channels simultaneously, offering a tremendous increase in the output. However, these systems are blind; i.e., the users do not have control over the cells they are measuring. While that does not cause a major problem with cultured cells, which are grown in culture dishes as monolayers, being exposed to the nutrients homogenously; therefore, showing uniform characteristics, isolated (primary) cells may have more cellto-cell variations due to the three-dimensional structure of the tissue they are detached from and consequent concentration gradients in nutrients and growth factors. Planar systems do not work well with large cells such as cardiac myocytes.

Femtosecond lasers have previously been shown to create membrane depolarization [21], by forming sub-micron holes on cellular membranes, without harming the cells in general; yet, the membrane potential and its alterations due to the laser application were measured using a conventional patch-clamp system, rather than suggesting an alternative to the patchclamp pipette. Besides, other nanosurgery applications such as femtosecond laser-assisted transfection [22] or cellular sucrose deposition [23], which barely create an opportunity for largescale applications, laser-induced membrane depolarization by itself also does not promise a wide applicability, because it still requires a conventional patch-clamp setup. In addition to membrane depolarization, ultrafast laser pulses are also shown to create micron-sized holes in polymer films in a different study [24]. Here, we suggest that combining these two separate works of membrane depolarization and polymer film puncture, femtosecond laser pulses could be used to measure membrane currents throughout the cells, as a new technique for electrophysiological measurements of cells, a laser-assisted cellular electrophysiology measurement (LACEM) system. Omitting the micropipettes, this method could facilitate electrophysiological measurements substantially.

\section{EXPERIMENTAL DESIGN}

A cell bath is prepared by separating two cylinder-shaped containers by attaching a thin Mylar film in between. Each cylinder has its own liquid inlet and outlet, and electrode insertion sealed with silicone. Here, the below part is the

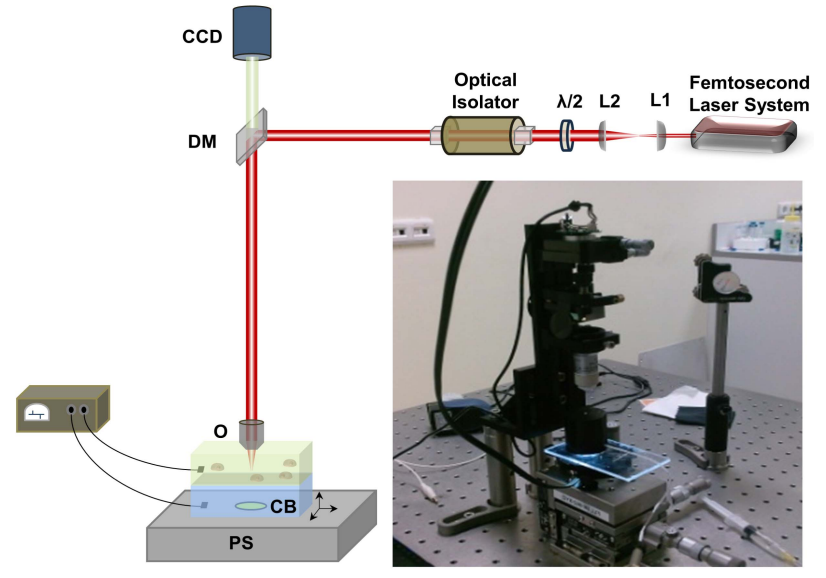

Fig. 1. Experimental setup. The Ti:Sapphire femtosecond laser is coupled to a custom-built microscope from its side-port. Laser illumination passes through a beam expander lens system, a half-wave waveplate, an optical isolator, and it is reflected towards the sample using a dichroic mirror (DM). It is focused using a 100x water-immersion microscope objective $(\mathrm{O})$, which is dipped into the bath solution. The image is collected using a charge-coupled device (CCD) camera. In the cell bath (CB) the upper compartment has a physiological solution, and the other contains an electrode solution. The electrodes are connected to a miniature patch-clamp amplifier. A positioning system (PS) is intended to be used to facilitate the delivery of cells to the femtosecond laser pulse. The picture of the actual system is also given in the figure.

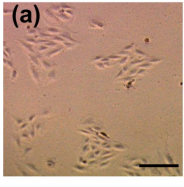

(d)

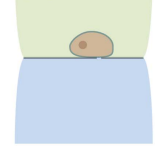

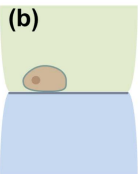

(e)

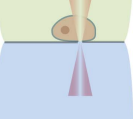

(c)

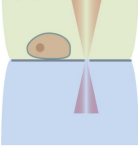

(f)

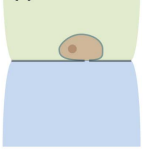

Fig. 2. Laser-assisted electrophysiological measurement. After the H9c2 cells grown in culture (a) (Scale bar $=200$ microns) are harvested and placed into the upper compartment of the cell bath, and they settle down on the polymer film (b), a femtosecond laser pulse is used to punch the membrane separating the two baths, nearby a cell (c), and the cell is brought on top of the hole by applying a negative pressure using suction (d). Afterwards, another laser pulse is used to punch a hole in the cell membrane (e) This causes the cell membrane to be exposed to the solution in the lower compartment, in a similar manner to a whole-cell patch-clamp configuration (f).

electrode solution, while the above part is the bath solution. $\mathrm{H} 9 \mathrm{c} 2$ rat cardio myoblast cells were used in patch-clamp experiments because of their electrically excitable nature [18]. For this, a diluted cell culture suspension is dropped onto the Mylar film and the electrodes are connected to a Tecella Pico miniature patch-clamp amplifier. An upright microscope is used to observe the cells and the experiment is conducted after the cells are settled down. The schematic representation of the experimental set-up is given in Fig. 1. Femtosecond laser pulses generated from a Ti:Sapphire laser is coupled to the home-made microscope from its side-port. The laser operates at $800 \mathrm{~nm}$, which is a wavelength that has minimal harm on living tissues [25]. The tail of the laser emission is visible to the human eye, which is used for alignment of the laser beam. 

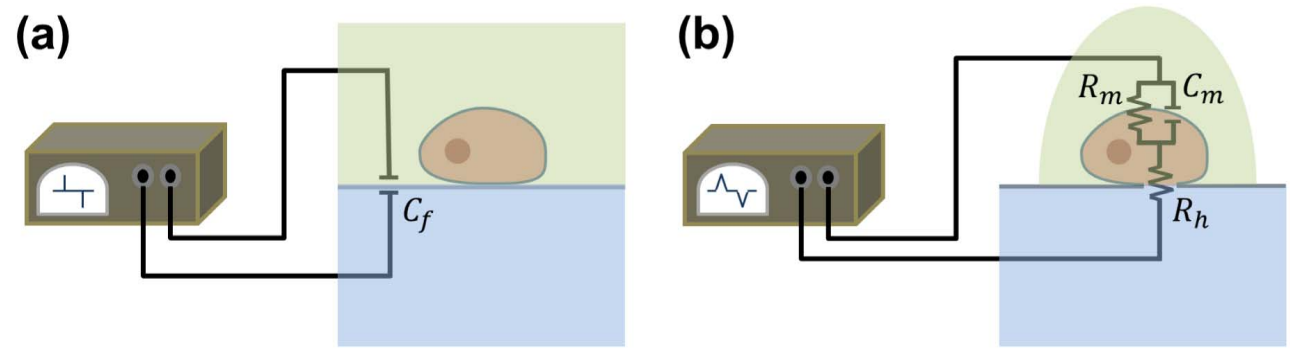

Fig. 3. Electrical analogue of the open cell using femtosecond laser. Before laser application, two electrodes are electrically isolated from each other, enabling observations of only short capacitive currents through the polymer membrane $\left(C_{f}\right)$ (a). After the laser treatments and the liquid removal except the volume around the cell to reduce the $C_{f}$, the cell is now an RC circuit, in a similar fashion to patch-clamp experiments. The major electrical components are the hole and membrane resistances $\left(R_{h}\right.$ and $R_{m}$, respectively), and the membrane capacitance $\left(C_{m}\right)$, which is significantly lower than the $C_{f}$. Under these circumstances, membrane currents through the ion channels, caused by a voltage pulse become obvious, which could be observed using a patch-clamp amplifier (b).

(a)
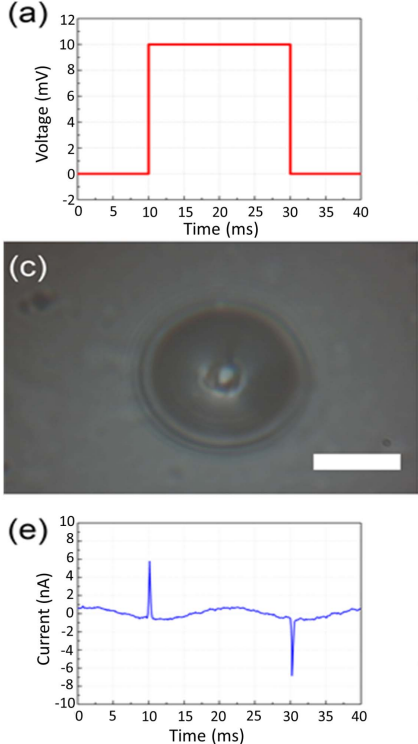
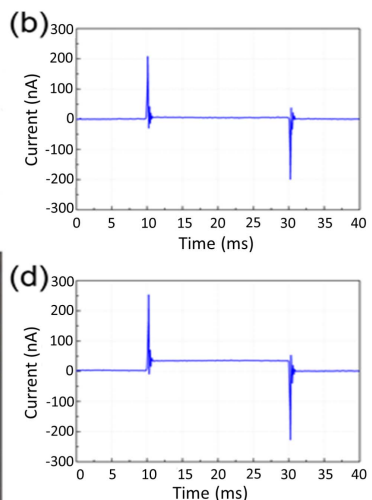

(f)

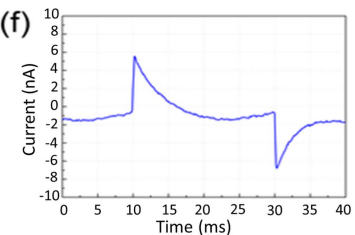

Fig. 4. Experimental demonstration of laser-assisted cellular electrophysiology measurements. A voltage pulse of $20 \mathrm{~ms}$ is used to probe the membrane currents (a). Initially, there is only the capacitive current present (b). When a hole on the Mylar membrane is formed (c) (Scale bar $=1$ micron), the corresponding current through the hole is observed (d). After the cell is sucked on top of the hole (e), which corresponds to the condition of Fig. 2(d), a hole on the cell is formed, revealing electric currents through voltagegated channels of $\mathrm{H} 2 \mathrm{c} 9$ cells (f). The liquid on the upper compartment, i.e., intracellular bath solution, is removed after sucking of the cell, refilled, and removed again, to reduce the effect of the capacitive currents. This is the electrical response depicted in Fig. 3 (b).

\section{RESULTS AND DISCUSSION}

At the beginning of the experiment, the electrode potential difference is a function of the resistivity and capacitance of the Mylar film. Then, the laser is first focused on the Mylar film just beneath, or beside the target cell, using a low laser power, and a femtosecond pulse is applied to form a hole in the Mylar film. Afterwards, a negative pressure is created by applying suction, which seals the cell on top of the newly formed hole. Finally, another pulse is applied to the same position, which opens a hole this time on the cell membrane and opens the cell electrically. A schematic image of the cell on the set-up is presented in Fig. 2.

After the cell is sealed onto the hole formed on the Mylar film, and another hole is formed on the cell membrane, the cell becomes electrically analogous to an RC circuit, in a similar

fashion to the patch-clamp using a micropipette (Fig. 3). Here, the examined electric current is formed by the ions passing through the ion channels of the protein structure embedded in the cell membrane. The membrane resistance varies as the ion channels open and close. There is a small capacitance of the cell membrane, and since the ion flow through the hole at the cell membrane will be compromised by the small dimension of it, the hole constitutes a second resistance, which has a fixed value. Before the hole on the Mylar film is formed, a voltage pulse of $20 \mathrm{~ms}$ (Fig. 4a) only creates an instantaneous capacitive current (Fig. 4b), while a hole opened on the Mylar film by the femtosecond laser (Fig. 4c) creates a constant current (Fig. 4d) with the same voltage pulse. After the suction is performed and the cell is placed on top of the hole (Fig. 4e), this current is subsided, which is an indication of a good seal, with similar temporal characteristics to the capacitive currents observed with micropipettes that have a comparable capillary size. In a similar fashion with the micropipettes, the smaller the capacitive current, the better the seal is, and the suction is enough for that. Finally, the cell membrane is opened again with another femtosecond pulse, which enables the observation of currents through the voltage-dependent channels of H9c2 cells (Fig. 4f). Here, it is important to note that, after the final step of membrane opening, most of the liquid on the top compartment of the measurement chamber should be removed, since the capacitance is:

$$
C_{f}=\epsilon_{f} \frac{A_{f}}{d_{f}}
$$

where $C_{f}$ is the capacitance of the polymer film, $\epsilon_{f}$ is the electric permittivity of the Mylar, $A_{f}$ is the area of film between the compartments, and $d_{f}$ is the film thickness. The reduction of the area between the compartments minimizes the capacitive currents. On the other hand, removal of the liquid creates a lens effect, which might affect the focusing of the femtosecond laser. Thus, it is applied as the last step before the measurement.

\section{CONCLUSION}

In this study, we demonstrate a convenient method for exploring the cellular membrane currents, by eliminating the need for using glass capillary tubes. This technique also enables selecting the cell that would be investigated. Different patch configurations, such as cell-attach, whole-cell, insideout, and outside-in could be mimicked by changing the 
initial hole size on the Mylar film, and constituents of the electrode and bath solutions. For instance, opening a hole on the Mylar membrane, and placing the cell on the hole by suction would be cell-attach configuration. If the hole is large, it would be whole-cell configuration. If there would be a small hole, by changing the constituents of the electrode and bath solutions, inside-out and outside-in configurations would be attained. The hole size could be adjusted by changing the focus of the femtosecond laser [24].

LACEM system is flexible and suitable for being developed further into a widely applicable technique. Particularly the design of the cell bath chamber could also be further optimized without changing the operational principle. The only prerequisite is using an ultrashort pulse laser, operating at a wavelength that does not interact with a living tissue. Alternative femtosecond lasers, for instance [26], can replace Ti:Sapphire laser systems, which are bulky and expensive. The patch-clamp amplifier we used in this study could also be superseded by better equipment; yet, they would not be as cost-effective as described in this work. Our major aim was to demonstrate an example of a facile and low-cost electrophysiological measurement system, which we believe that we provide sufficient experimental evidence.

Further studies are necessary to fully characterize our system, particularly using different types of cells under different protocols. On the other hand, this study is, to our knowledge, still the very first demonstration of a laser-assisted electrophysiological measurement system, providing clear insights regarding its applicability. It is among our future plans to continue the investigation related to this study and explore the possibilities in terms of transforming this knowledge into a product that would be well-established and widely accessible.

As a conclusion, we have successfully developed a facile system, using femtosecond laser nanosurgery, to measure the activity of ion channels, in a similar fashion to patch-clamp recording, the gold standard in electrophysiology, paving the way for higher throughput electrophysiological measurements by providing a measurement scheme that does not use glass micropipettes, thus overcoming the biggest complexity in patch-clamp recording.

\section{ACKNOWLEDGMENT}

The authors would like to thank Prof. Ümit Bağrıaçık from Gazi University for providing $\mathrm{H} 9 \mathrm{c} 2$ cell line and Nanobiotechnology Group, Bilkent University, UNAM for their support in cell culture maintenance. A. A. Seymen and E. Özgür declare financial interest regarding LACEM through E-A Teknoloji LLC.

\section{REFERENCES}

[1] B. Alberts, A. Johnson, J. Lewis, M. Raff, K. Roberts, and P. Walter, Molecular Biology of the Cell, 4th ed. New York, NY, USA: Garland Science, 2002.

[2] A. Krogh, B. Larsson, G. von Heijne, and E. L. L. Sonnhammer, "Predicting transmembrane protein topology with a hidden Markov model: Application to complete genomes," J. Mol. Biol., vol. 305, no. 3, pp. 567-580, 2001
[3] J. P. Overington, B. Al-Lazikani, and A. L. Hopkins, "How many drug targets are there," Nature Rev. Drug Discovery, vol. 5, no. 12, pp. 993-996, Dec. 2006.

[4] M. Edidin, "Lipids on the frontier: A century of cell-membrane bilayers," Nature Rev. Mol. Cell Biol., vol. 4, no. 5, pp. 414-418, May 2003.

[5] B. Hille, Ion Channels of Excitable Membranes, 3rd ed. Sunderland, MA, USA: Sinauer Associates, 2011

[6] C. D. Strader, T. M. Fong, M. R. Tota, D. Underwood, and R. A. F. Dixon, "Structure and function of G protein-coupled receptors," Annu. Rev. Biochem., vol. 63, no. 1, pp. 101-132, 1994.

[7] L. S. Musil, B. A. Cunningham, G. M. Edelman, and D. A. Goodenough, "Differential phosphorylation of the gap junction protein connexin 43 in junctional communication-competent and-deficient cell lines," J. Cell Biol., vol. 111, no. 5, pp. 2077-2088, Nov. 1990.

[8] B. Geiger, A. Bershadsky, R. Pankov, and K. M. Yamada, "Transmembrane extracellular matrix-cytoskeleton crosstalk," Nat. Rev. Mol. Cell Biol., vol. 2, no. 11, pp. 793-805, Nov. 2001.

[9] W. A. Catterall, "Structure and function of voltage-gated ion channels," Annu. Rev. Biochem., vol. 64, no. 1, pp. 493-531, Jun. 1995.

[10] G. R. Dubyak and C. El-Moatassim, "Signal transduction via P2 purinergic receptors for extracellular ATP and other nucleotides," Amer. J. Physiol.-Cell Physiol., vol. 265, no. 3, pp. C577-C606, Sep. 1993.

[11] R. Inoue et al., "Synergistic activation of vascular TRPC6 channel by receptor and mechanical stimulation via phospholipase C/diacylglycerol and phospholipase A(2)/ $\omega$-hydroxylase/20-HETE pathways," Circulat. Res., vol. 104, no. 12, pp. 1399-1409, Jun. 2009.

[12] D. D. McKemy, W. M. Neuhausser, and D. Julius, "Identification of a cold receptor reveals a general role for TRP channels in thermosensation," Nature, vol. 416, no. 6876, pp. 52-58, Mar. 2002.

[13] G. Nagel et al., "Channelrhodopsin-2, a directly light-gated cationselective membrane channel," Proc. Nat. Acad. Sci. USA, vol. 100, no. 24, pp. 13940-13945, Nov. 2003.

[14] W. N. Green and O. S. Andersen, "Surface charges and ion channel function," Аnnu. Rev. Physiol., vol. 53, no. 1, pp. 341-359, Oct. 1991.

[15] G. Cavelier, "Possible role of surface electrochemical electron-transfer and semiconductor charge transport processes in ion channel function," Bioelectrochem. Bioenergetics, vol. 40, no. 2, pp. 197-213, Aug. 1996.

[16] W. Wang, M. Watanabe, T. Nakamura, Y. Kudo, and R. Ochi, "Properties and expression of $\mathrm{Ca}^{2+}$-activated $\mathrm{K}^{+}$channels in $\mathrm{H} 9 \mathrm{c} 2$ cells derived from rat ventricle," Amer. J. Physiol.-Heart Circulatory Physiol., vol. 276, no. 5, pp. H1559-H1566, May 1999.

[17] J. Hescheler, R. Meyer, S. Plant, D. Krautwurst, W. Rosenthal, and G. Schultz, "Morphological, biochemical, and electrophysiological characterization of a clonal cell (H9c2) line from rat heart," Circulat. Res., vol. 69, no. 6, pp. 1476-1486, Dec. 1991.

[18] K. R. Sipido and E. Marban, "L-type calcium channels, potassium channels, and novel nonspecific cation channels in a clonal muscle cell line derived from embryonic rat ventricle," Circulat. Res., vol. 69, no. 6, pp. 1487-1499, Dec. 1991.

[19] E. Neher and B. Sakmann, "Single-channel currents recorded from membrane of denervated frog muscle fibres," Nature, vol. 260, no. 5554, pp. 799-802, Apr. 1976.

[20] N. Fertig, R. H. Blick, and J. C. Behrends, "Whole cell patch clamp recording performed on a planar glass chip," Biophys. J., vol. 82, no. 6, pp. 3056-3062, Jun. 2002.

[21] J. Ando, N. I. Smith, K. Fujita, and S. Kawata, "Photogeneration of membrane potential hyperpolarization and depolarization in non-excitable cells," Eur. Biophys. J., vol. 38, no. 2, pp. 255-262, Feb. 2009.

[22] D. Stevenson et al., "Femtosecond optical transfection of cells: Viability and efficiency," Opt. Exp., vol. 14, no. 16, pp. 7125-7133, Aug. 2006.

[23] V. Kohli, J. P. Acker, and A. Y. Elezzabi, "Reversible permeabilization using high-intensity femtosecond laser pulses: Applications to biopreservation," Biotechnol. Bioeng., vol. 92, no. 7, pp. 889-899, Dec. 2005.

[24] R. Nielson and J. B. Shear, "Parallel chemical dosing of subcellular targets," Anal. Chem., vol. 78, no. 17, pp. 5987-5993, Sep. 2006.

[25] Q. Peng et al., "Lasers in medicine," Rep. Prog. Phys., vol. 71, no. 5, May 2008, Art. no. 056701.

[26] U. Demirbas, A. Sennaroglu, A. Benedick, A. Siddiqui, F. X. Kartner, and J. G. Fujimoto, "Diode-pumped, high-average-power femtosecond $\mathrm{Cr}^{3+}$ : LiCAF laser," Opt. Lett., vol. 32, no. 22, pp. 3309-3311, Nov. 2007. 